\title{
at - Automatisierungstechnik
}

Theoretische Grundlagen, Methoden, Anwendungen

46. Jahrgang 1998 - Heft 11 - Seite 503-554

\section{Schwerpunktthema:}

System- und Regelungstechnik in der Medizin

\section{EDITORIAL}

G. Schmidt, J. Werner

System- und Regelungstechnik in der Medizin

\section{ANWENDUNGSAUFSATZ · APPLICATION PAPER}

R. Riener, T. Fuhr, G. Schmidt, J. Quintern

Entwurf einer geregelten Neuroprothese für das Aufstehen unter Berücksichtigung der intakten Willkürmotorik

The design of a closed-loop neuroprosthesis incorporating intact voluntary effort for standing-up

\section{J. Werner, M. Hexamer, K. Hoeland, M. Meine}

Sensorgesteuerte Herzschrittmacher-Systeme

Sensor-controlled heart pacemaker systems

\section{Nix, F. Förster, R. Kaufmann, H. Reul, G. Rau}

Ansätze eines fuzzy-basierten Regelungssystems für pulsatile elektromechanische Herzunterstützungs- und Herzersatzsysteme

Fuzzy control concept for pulsatile electromechanical heart support and replace systems

S. Leonhardt, S. Böhm, B. Lachmann

Optimierung der Beatmung beim akuten Lungenversagen durch Identifikation physiologischer Kenngrößen

Optimal Artificial Ventilation by Identification of Physiological Parameters

\section{R. E. Löser}

Medizintechnische Geräte - Eine Herausforderung an die Automatisierungstechnik

Medical Devices - A Challenge for Automatisation Technique

\section{W. Düchting}

Computermodelle zur Optimierung der Strahlentherapie

Computer models for optimizing radiation therapy

\section{RUBRIKEN · COLUMNS}

Aus der Arbeit der GMA - GMA activities

Dissertationen - Dissertations
Peter Haase

Das Jahr 2000 in der EDV

Bewältigung des Jahr-2000-Problems

in Ihrem Unternehmen

1. Auflage 1998. 250 Seiten

DM 98,- $/$ ÖS $715,-1$ sFr $85,-$

ISBN 3-486-24737-9

- Subskriptionspreis bis 30.04.1998 DM 78,- / öS 569,- / sFr 68,-

Computer werden von Programmen gesteuert, die in vielen Fällen Datumswerte ab dem Jahr 2000 nicht fehlerfrei verarbeiten können.

Das ist das Jahr-2000-Problem.

Dieses Buch hilft, Gefahrenquellen zu erkennen und die richtigen Maßnahmen einzuleiten.

Zum ersten Mal wird der Themenkomplex umfassend dargestellt. Folgende Aspekte werden ausführlich behandelt:

- Das Management von Jahr-2000Projekten

- die Bestandsaufnahme und Analyse der Datenbestände

- produkt- und projektunabhängige Lösungswege

- Testverfahren zur Prüfung der Jahr2000-Fähigkeit

- die Auswahl von und der Umgang mit Lösungsanbietern

- die bilanzielle Bewertung von Software

- haftungsrechtliche Fragen

- und Versicherungen.

Um die Gefahren abzuwenden, die für eine Organisation durch das Jahr-2000Problem entstehen können, sollten sich sowohl kaufmännisches und technisches Management als auch die Mitarbeiter der Unternehmen intensiv mit den angesprochenen Themen auseinandersetzen.

\section{Bestellschein}

Bitte senden Sie Ihre Bestellung an Ihre Buchhandlung oder an:

R. Oldenbourg Verlag

Rosenheimer Straße 145,

81671 München

Fax 089-45051-204

Ich (Wir) bestelle(n)

Expl. Haase

Das Jahr 2000 in der EDV ISBN 3-486-24737-9

Name und Anschrith

Datum/Unterschrift 